\title{
Improved technique for quasar composite spectra construction
}

\section{G. Ivashchenko*}

Astronomical Observatory, Taras Shevchenko National University of Kyiv, Kyiv, Ukraine

E-mail: g.ivashchenko@gmail.com

\section{O. Sergijenko}

Astronomical Observatory, Ivan Franko National University of Lviv, Lviv, Ukraine

E-mail: olka@astro.franko.lviv.ua

\section{O. Torbaniuk}

Faculty of Physics, Taras Shevchenko National University of Kyiv, Kyiv, Ukraine

E-mail: el.torbaniuk@gmail.com

We present a new approach to the composite spectra construction based on stacking spectra with similar slopes $\alpha_{\lambda}$ within the wavelength range redward of Ly $\alpha$ emission line, which allows to reduce the typical noise. With the help of this technique a detailed study of the Hi Ly $\alpha$-forest region $\left(\lambda_{\text {rest }} \approx 1050-1200 \AA\right.$ ) of the own sample of 3439 medium-resolution quasar spectra from SDSS DR7 was performed. More than 14 lines were found within this wavelength range, three of which were known from previous studies of quasar composite spectra from SDSS and some others were known in composite spectra from space-based telescopes or high-resolution spectra of individual quasars from ground-based telescopes.

VI European Summer School on Experimental Nuclear Astrophysics, ENAS 6

September 18-27, 2011

Acireale Italy

*Speaker. 


\section{Introduction}

Composite spectra of extragalactic objects play an important role in studing their different properties, because they allow to reduce signal-to-noise ratio and reveal tiny features unseen in individual spectra of low or medium resolution. In the case of quasars, which have similar individual spectra, the composite spectra are used for redshift determination (mainly in automatic redshift surveys), for calculation of quasar color-indices (used for photometric selection of quasar candidates) and in the studies of the Ly $\alpha$ forest. The last one is a 'forest' of absorption Ly $\alpha$ lines in quasar spectra originated in intergalactic medium. It traces the thermal and radiative history of the Universe, as well as the evolution of underlying matter distribution over a wide range of scales and redshifts, thanks to the relation between the Ly $\alpha$ opacity of the intergalactic $\mathrm{H}$ I and its density and other physical parameters. Fluctuations of transmission $F$ (a measure of opacity) provide an information about the density fluctuations on the smallest scales available today for observations (see e.g. $[5,13]$ ), which in their turn give constraints of the physical properties of the dark matter particles, e. g. sterile neutrino masses [3].

The composite spectra of quasars in ultraviolet-optical band were compiled for a wide set of quasar samples $[4,7,12,16]$. The composite spectra were also used in Ly $\alpha$-forest studies in $[1,6,11]$. One of the main sources of uncertainties during the generation of the composite spectra of quasars is the difference of the quasar spectra in the slopes $\alpha_{\lambda}$ of the continuum level in the red part of UV-optical bump, despite the strong similarity between the quasar spectra. Thus in the present work we tried to generate the composite spectra from the samples of spectra with the $\operatorname{similar} \alpha_{\lambda}$.

\section{The data and composite spectra generation}

Our sample was taken from the public available release of the sky-residual subtracted spectra for the Sloan Digital Sky Survey Data Release 7 (SDSS DR7) [17] with redshifts from [8]. All the quasar spectra (15154 objects) from this sample with redshifts within the range 2.3-4.6 and redshift determination confidence level $>0.9$ were firstly selected and examined by eye. The spectra with low signal-to-noise ratio, broad absorption lines (BAL) and Damped Ly $\alpha$ (DLA) systems were excluded during this examination. This selection reduced the number of spectra to 4779 . The (cosmological) redshift distribution of the sample is presented in Fig. 1 (left). The cosmological redshift is a shift of the spectral energy distribution of objects toward longer wavelengths due to the Universe expansion. It is related to the distance according to accepted cosmological model. Within the common spatially flat $\Lambda \mathrm{CDM}$ model the redshift range shown in Fig. 1 corresponds to the distance range of about $20-40 \mathrm{Gpc}$.

Generating composite spectra of quasars includes three steps: (i) normalization of each spectrum, (ii) setting each spectrum to the rest frame and (iii) calculation of the mean spectrum. Before this, all the spectra were smoothed with a simple moving average over 3 points and regions with known calibration problems were removed. The mean flux in all pixels within the rest wavelength range $1450-1470 \AA$ has been used as a normalization constant $A$ because this range is usually considered to be free of obvious emission and absorption. In order to to reduce possible uncertainties, we used only spectra with the rms of the normalization constant $A$ less than $15 \%$; the number of 

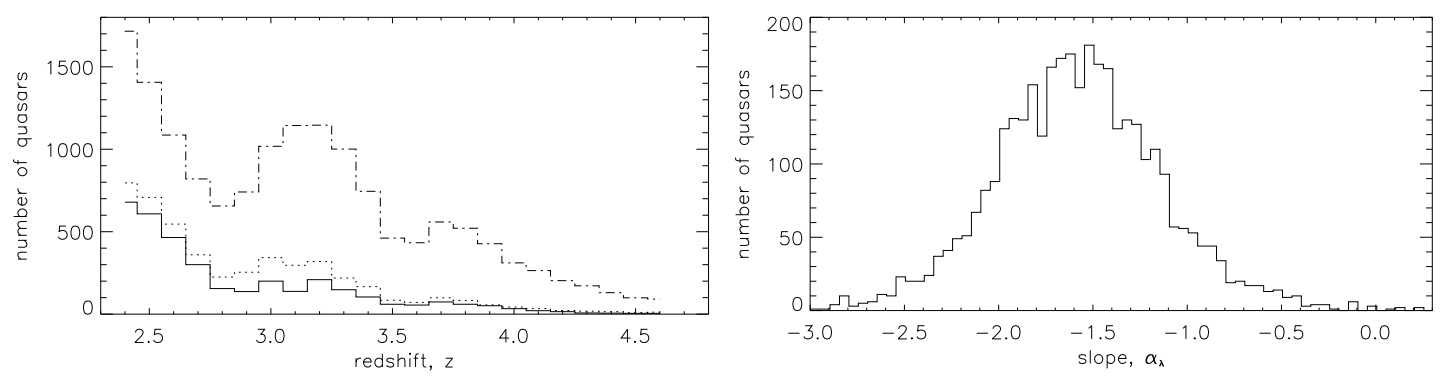

Figure 1: Left: Redshift distribution of initial sample (dash-dot), all visually selected (dotted) for the present study and those with the rms of the normalization constant $A$ less than $15 \%$ (solid). Right: the distribution of the true continuum slopes redward of $\operatorname{Ly} \alpha$ emission line.

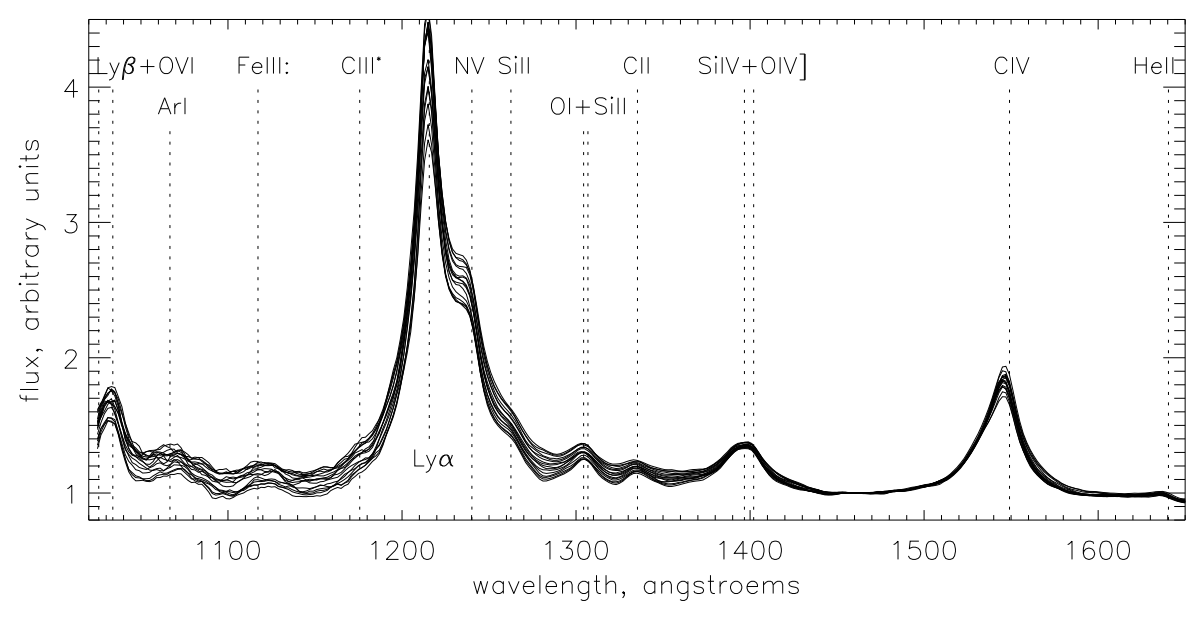

Figure 2: Composite spectra of 16 subsamples within 1025-1650 $\mathrm{A}$. The upper spectrum has the steeper true continuum redward of $\operatorname{Ly} \alpha$ emission line.

them is 3493 and their redshift distribution is presented in Fig. 1 (left as a solid line). Considering the true continuum of the quasar spectra redward of Ly $\alpha$ emission line to be a power-low $\sim \lambda^{\alpha_{\lambda}}$, we calculated its slope $\alpha_{\lambda}$ for each individual spectrum within the range between Ly $\alpha$ and $\operatorname{CIV}(1549.0 \AA)$ emission lines. The distribution of the obtained slopes is shown in Fig. 1 (right).

Using the obtained values of $\alpha_{\lambda}$, we selected 16 subsamples each of 200 spectra with $\alpha_{\lambda}$ closest to $-0.7-k \cdot 0.1, k=0 . .15$. Then dividing each $j$-th selected spectrum onto its normalization constant $A^{j}$, rebinning them with $\Delta \lambda_{\text {rest }}=2 \AA$ and stacking the spectra into the rest frame, we obtained the mean arithmetic composite spectra. These spectra within 1025-1650 ̊ are shown in Fig. 2. The dashed lines indicate the laboratory wavelengths of the lines identified in [16].

\section{The technique}

The mean quasar continuum $\bar{C}\left(\lambda_{\text {rest }}\right)$ within the Ly $\alpha$ forest region can be presented as a sum of the true continuum, which is usually assumed to have a power-low form [1], and the emission lines which are fitted well with Gaussian profile. But unlike the common treatment we considered the 'pure' continuum of this wavelength region as a constant (such treatment within the region only $\approx 150 \AA$ wide seems to be precise enough). Hence the fitting formula for the product of the mean 
transmission and continuum $f(\lambda)=\bar{F} \bar{C}\left(\lambda_{\text {rest }}\right)$ is the following:

$$
f(\lambda)=b+\sum_{k} a_{k} \exp \left[-\frac{\left(\lambda-\lambda_{k}^{0}\right)^{2}}{2 w_{k}^{2}}\right] .
$$

Here $\lambda$ is the rest frame wavelength, $a_{k}, \lambda_{k}, w_{k}$ are the amplitudes, the central wavelengths and FWHM (up to factor of $\sqrt{2}$ ) of each $k$-th emission feature, and $b$ is the constant describing continuum. Because we are interested more in parameters of the lines rather than the global fit of all Ly $\alpha$ forest region with one function, we splitted this region onto three parts corresponding to each emission feature (multiplet) and fitted each part separately. The fitting procedure was conducted in the following two steps: (i) using the IDL lmfit subroutine for each multiplet (with central wavelengths around 1070, 1120 and $1215 \AA$ ) the best fit model in a form of (3.1) was found; (ii) the central wavelengths $\lambda^{0}$ obtained at the first step were fixed and the best fit values of other parameters $\left\{b, a_{k}, w_{k}\right\}$ with the $1 \sigma$ errors from the extremal values of the $\mathrm{N}$-dimensional distribution were calculated by the Markov Chain Monte Carlo (MCMC) method using CosmoMC package [10] as a generic sampler (with the values of $\left\{b, a_{k}, w_{k}\right\}$ obtained previously as starting values). The MCMC technique has been chosen as it is fast and accurate method of exploration of high-dimensional parameter spaces. In each case we generated 8 chains which have converged to $R-1<0.0075$.

\section{Results and conclusions}

As an example the composite spectrum with $\alpha_{\lambda}=-0.92$ together with best fits and found lines are presented in Fig. 3. The central wavelengths of all the lines found in composite spectra are presented in Table 1. Here 'tent.' means tentative identification by eye (which was not included in fit). Despite of variance in central wavelengths and the number of lines in different spectra we tried to systematize them. In most cases, except the multiplet around $1120 \AA$, the lines seem to be the same in all spectra, but due to the difference in their FWHM in one spectrum two given lines are resolved, while in another spectrum they are blended and appear to be fitted better with one Gaussian. The values in brackets mean such dublets fitted by one Gaussian in case of triplets. The line identifications were taken from the papers on composite UV spectra of quasars obtained with HST and FUSE missions [12, 14, 18], high-resolution spectra of single quasars Ton34 [2] and PHL1811 [9], and also composite spectra of quasars from optical ground Kast [15] and SDSS [16] surveys. The lines which were not identified with known lines from these papers are labelled with $\mathrm{X}_{1}-\mathrm{X}_{10}$. The obtained values of the line parameters will be presented elsewhere.

The proposed approach can be applied for generation of new templates for more precise quasar redshift measurements with the common cross-correlation technique used in redshift surveys, more precise theoretical determination of K-correction and color-indices, as far as for determination of continuum and mean transmission in $\operatorname{Ly} \alpha$ forest studies.

\section{Acknowledgements}

This work has been supported by Swiss National Science Foundation (SCOPES grant No 128040). The authors are thankful to the Sloan Digital Sky Survey team and also acknowledge the usage of CosmoMC package. 


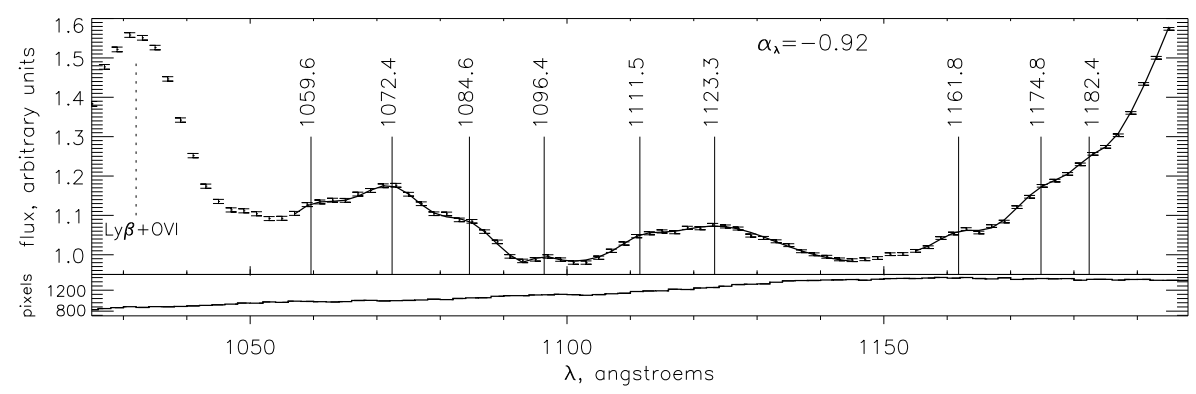

Figure 3: Composite spectrum with $\alpha_{\lambda}=-0.92$.

\section{References}

[1] M. Bernardi, et al., A Feature at $z \sim 3.2$ in the Evolution of the Ly $\alpha$ Forest Optical Depth, AJ 125, 32 [astro-ph/0206293].

[2] L. Binette \& Y. Krongold, The emission-line spectrum of the UV deficient quasar Ton 34: evidence of shock excitation?, AAP 477, 413 [0710.0324].

[3] A. Boyarsky, J. Lesgourgues, O. Ruchayskiy \& M. Viel, Lyman- $\alpha$ constraints on warm and on warm-plus-cold dark matter models, JCAP 05012 [0812 . 0 010].

[4] M. S. Brotherton, H. D. Tran, R. H. Becker, M. D. Gregg, S. A. Laurent-Muehleisen \& R. L. White, Composite Spectra from the FIRST Bright Quasar Survey, ApJ, 546, 775 [astro-ph/ 0008396 ].

[5] M. Cappetta, V. D’Odorico, S. Cristiani, F. Saitta \& M. Viel, High-resolution spectroscopy of the 3D cosmic web with close QSO groups, MNRAS 4071290 [1 004 . 0221].

[6] V. Desjacques, A. Nusser \& R. K. Sheth, The probability distribution function of the Lyman $\alpha$ transmitted flux from a sample of Sloan Digital Sky Survey quasars, MNRAS 374, 206 [astro-ph/0608167].

[7] P. J. Francis, P. C. Hewett, C. B. Foltz, F. H. Chaffee, R. J. Weymann \& S. L. Morris, A high signal-to-noise ratio composite quasar spectrum, ApJ 373, 465.

[8] P. C. Hewett \& V. Wild, Improved redshifts for SDSS quasar spectra, MNRAS 405, 2302 [1003.3017].

[9] K. M. Leighly, J. P. Halpern, E. B. Jenkins \& D. Casebeer, The Intrinsically X-Ray-weak Quasar PHL 1811. II. Optical and UV Spectra and Analysis, ApJS 173, 1 [0705.0940].

[10] A. Lewis \& S. Bridle, Cosmological parameters from CMB and other data: A Monte Carlo approach, Phys. Rev. D 66103511 [astro-ph/0205436], http://cosmologist.info/cosmomc

[11] G. Polinovskyi \& M. Malygin, Mean Transmitted Flux in the Ly $\alpha$ Forest from a Sample of 2QZ Quasars, in WDS'10 Proceedings of Contributed Papers: Part III - Physics, 163 [1106 . 2560]

[12] J. Scott, G. Kriss, M. Brotherton, R. Green, J. Hutchings, J. Shull \& W. Zheng, A Composite Extreme Ultraviolet QSO Spectrum from FUSE, in AGN Physics with the Sloan Digital Sky Survey, ASPCS 311, 31 [astro-ph/0403662].

[13] A. Slosar et al., The Lyman- $\alpha$ forest in three dimensions: measurements of large scale flux correlations from BOSS 1st-year data, JCAP 09001 [1104 . 524 4].

[14] R. C. Telfer, W. Zheng, G. A. Kriss \& A. F. Davidsen, The Rest-Frame Extreme-Ultraviolet Spectral Properties of Quasi-stellar Objects, ApJ 565, 773 [astro-ph/ 0109531 ]. 
[15] D. Tytler, J. M. O’Meara, N. Suzuki, D. Kirkman, D. Lubin \& A. Orin, The Kast Ground-based Ultraviolet Spectral Survey of 79 Quasi-stellar Objects at Redshift 2 for Ly $\alpha$ Forest and Metal Absorption, AJ 128, 1058 [astro-ph/ 0405051 ].

[16] D. E. Vanden Berk et al., Composite Quasar Spectra from the Sloan Digital Sky Survey, AJ 122, 549 [astro-ph/0105231].

[17] V. Wild \& P. C. Hewett, Peering through the OH Forest: public release of sky-residual subtracted spectra for SDSS DR7, 1010.2500.

[18] W. Zheng, G. A. Kriss, R. C. Telfer, J. P. Grimes \& A. F. Davidsen, A Composite HST Spectrum of Quasars, ApJ 475, 469 [astro-ph/9608198]. 
Table 1. Emission lines found in spectra. Lines in brackets are dublets in case of triplets. Arrows stand for failed fit.

\begin{tabular}{|c|c|c|c|c|c|c|c|c|}
\hline & 1 & 2 & 3 & 4 & 5 & 6 & 0 & 8 \\
\hline$\alpha_{\lambda}$ & $\mathrm{X}_{1}$ & $\mathrm{X}_{2}+\mathrm{ArI}+\mathrm{X}_{3}$ & $\mathrm{NII}+\mathrm{X}_{4}$ & $\mathrm{X}_{5}+\mathrm{X}_{6}$ & FeIII-multiplet & $\mathrm{X}_{7}$ & $\mathrm{X}_{8}+\mathrm{CIII}^{*}+\mathrm{X}_{9}$ & $\mathrm{X}_{10}+\mathrm{Ly} \alpha$ \\
\hline-0.84 & tent. & $1058.4+[1071.8]$ & 1084.9 & 1096.3 & $1111.8+1125.2$ & & $1161.6+1173.9+1182.1$ & 1214.4 \\
\hline-0.92 & tent. & $1059.6+[1072.4]$ & 1084.6 & 1096.4 & $1111.5+1123.3$ & tent. & $1161.8+1174.8+1182.4$ & 1214.3 \\
\hline-0.99 & tent. & $1059.5+[1073.9]$ & 1086.1 & 1096.9 & $1118.3+1125.7+1134.4$ & tent. & $1161.4+[1176.5]$ & 1214.2 \\
\hline-1.02 & tent. & $1059.5+[1071.0]$ & 1089.3 & tent. & $1117.0+1126.2+1136.5$ & tent. & tent. $+1175.2+1185.3$ & 1214.7 \\
\hline-1.19 & tent. & tent. $+[1070.7]$ & 1087.6 & & $1115.2+1124.4+1136.2$ & 1152.3 & 1171.9 & 1214.3 \\
\hline-1.30 & $\rightarrow$ & $\rightarrow$ & $\rightarrow$ & 1100.9 & $1116.9+1125.0+1132.4$ & tent. & tent. $+[1174.2]$ & 1213.2 \\
\hline-1.42 & $\rightarrow$ & $1061.6+[1071.1]$ & $1079.3+1091.0$ & & $1108.4+1116.6+1127.0+1143.9$ & & Otent. $+[1172.1]$ & 1213.2 \\
\hline-1.42 & $\rightarrow$ & $1060.3+[1071.3]$ & 1080.8 & 1098.5 & $1111.5+1125.4+1141.0$ & tent. & $\quad 1172.5$ & 1213.8 \\
\hline-1.49 & $\rightarrow$ & tent. $+[1070.2]$ & 1086.0 & 1099.6 & 1122.6 & tent. & 1172.6 & 1213.4 \\
\hline-1.55 & $\rightarrow$ & $1056.3+[1071.7]$ & 1086.8 & 1099.6 & $1117.3+1127.4+1141.7$ & tent. & $1163.7+[1174.2]$ & 1213.8 \\
\hline-1.67 & $\rightarrow$ & $1058.1+[1070.4]$ & 1079.9 & & $1106.7+1115.7+1125.6$ & tent. & {$[1168.5]+1174.9$} & 1213.8 \\
\hline-1.83 & $\rightarrow$ & {$[1063.0]+1074.1$} & 1084.0 & & $1115.5+1123.8+1129.0$ & & tent. $+1172.9+1178.5$ & 1214.4 \\
\hline-1.86 & $\rightarrow$ & {$[1063.8]+1072.6$} & $1082.0+1087.5$ & 1103.3 & $1111.9+1124.3+1135.9$ & 1153.9 & $1164.1+[1172.7]$ & 1214.5 \\
\hline-1.99 & $\rightarrow$ & $\rightarrow$ & $\rightarrow$ & 1101.6 & $1116.2+1126.2+1135.5$ & tent. & 1172.0 & 1214.4 \\
\hline-2.00 & 1055.8 & {$[1066.6]+1072.5$} & 1083.2 & tent & $1108.3+1117.3+1125.5+$ tent. & $\leftarrow$ & $\leftarrow$ & $\leftarrow$ \\
\hline-2.12 & 1052.4 & $1060.6+[1071.4]$ & 1083.1 & tent. & $1111.3+1123.7+1140.1$ & 1155.5 & $1165.7+[1174.3]$ & 1214.1 \\
\hline
\end{tabular}

